\title{
Cooperative Guidance Law against Highly Maneuvering Target with Dynamic Surrounding Attack
}

\author{
Zhikai Wang, ${ }^{1}$ Wenxing Fu $\mathbb{D}^{2}{ }^{2}$ Yangwang Fang, ${ }^{2}$ Zihao $W u,{ }^{1}$ and Mingang Wang ${ }^{1}$ \\ ${ }^{1}$ School of Astronautics, Northwestern Polytechnical University, Xi'an, China \\ ${ }^{2}$ Unmanned System Research Institute, Northwestern Polytechnical University, Xi'an, China
}

Correspondence should be addressed to Wenxing Fu; wenxingfu@nwpu.edu.cn

Received 9 December 2020; Revised 15 March 2021; Accepted 29 March 2021; Published 19 April 2021

Academic Editor: Paolo Castaldi

Copyright (C) 2021 Zhikai Wang et al. This is an open access article distributed under the Creative Commons Attribution License, which permits unrestricted use, distribution, and reproduction in any medium, provided the original work is properly cited.

In this paper, a new dynamic surrounding attack cooperative guidance law against highly maneuvering target based on decoupled model is proposed. First, a new dynamic surrounding guidance strategy is proposed, and virtual targets are introduced to establish the cooperative guidance model for dynamic surrounding attack. Second, a dynamic inverse method is used to decouple the cooperative guidance model, and extended state observers (ESOs) are introduced to estimate the disturbances caused by target maneuver. Then, the impact time and dynamic surrounding guidance (ITDSG) law against highly maneuvering target is designed based on a prescribed-time stable method and the decoupled model. Finally, numerical simulations are performed to illustrate the superiority and effectiveness of the proposed ITDSG.

\section{Introduction}

Cooperative guidance laws for multimissiles have captured the interest of many researchers since the seminal work by I.S. Jeon first appeared [1]. Cooperative guidance law against a static or low-speed target has been extensively studied over the past decades $[2,3]$. The early cooperative guidance laws mainly focused on the cooperative proportional navigation $(\mathrm{CPN})$ proposed by Tahk et.al. $[4,5]$, which were used to solve the problems of antiship missiles attacking the ship simultaneously. In fact, the CPN is not a true sense of cooperative guidance law, because it does not consider the information interaction between the missiles. Soon afterwards, the true sense of cooperative guidance laws including centralized [6] or distributed [7-9] cooperative guidance laws were studied.

Considering the communications between missiles, some researches have proposed cooperative guidance laws against static or constant velocity target. Zhou and Yang [10] used the range-to-go as a covariable to avoid the estimation of times-to-go and designed a cooperative guidance law against a static target with an undirected communication topology. Zhao et.al. $[7,11]$ designed a multimissile cooperative guid- ance law against single stationary target based on modelpredictive-control (MPC) and CPN.

Unlike the above scenarios, some researchers have focused on the research of cooperatively intercepting maneuvering target with multimissiles in recent years $[2,3]$. As is known to all, a missile can intercept a low maneuvering target (such as early warning aircraft) easily [12-15], but it is difficult for a missile to intercept a target with highly maneuverability (such as unmanned aerial vehicle). Thus, it is necessary to develop cooperative guidance law for multiple missiles against the highly maneuvering target. Some scholars have studied this problem in recent years. In [16], an ESO was introduced to estimate the unknown disturbance caused by the acceleration of target; furthermore, the cooperative guidance law was designed with the estimated disturbances and finite time consensus theory $[17,18]$. Based on the optimal control method, Nikusokhan and Nobahari [19] proposed a novel approach to derive a cooperative guidance law for two pursuers against one zero-lag evader with a random step maneuver. However, the shortage of the approach is that the maneuverability of the missile is supposed to be unlimited, which is impracticable in engineer application. Because of the limitation of the missiles' 
overload, by setting the virtual target to make each missile's joint reachable range cover different subintervals of target maneuvering range, the cooperative guidance law was designed in [20] based on BPN to ensure that at least one missile hits the target. For the three-dimensional (3D) terminal cooperative guidance law against the maneuvering target, Song et.al. [21,22] proposed two-direction cooperative guidance laws including acceleration commands in the LOS and normal LOS directions. The acceleration command in the LOS direction was developed to ensure all the missiles to hit the target simultaneously in a finite time; the normal acceleration command was designed to guarantee that LOS angular rate and LOS angle converge to the desired values. Unfortunately, the aforementioned methods did not consider the couplings between the LOS and normal LOS direction. Furthermore, the existing guidance strategies for surrounding attack require to set the desired LOS angles in advance, in fact this is a static strategy. However, in the actual combat environment, it is difficult to set the suitable LOS angles in advance.

In order to improve the effort of attacking a target, surrounding attack strategy must be considered. In the existing literatures, in order to achieve the surrounding attack, the term LOS angle constraint is added to the basic guidance law, so that the designed guidance law can ensure the missile to approach the target with a desired LOS angle. The guidance laws for single missile attacking single target with a LOS angle constraint have been extensively studied [23-25]. For the guidance problem of multimissile attack a target with time and LOS angle constraints, some researchers have studied them [26-29]. However, in the actual application, the relative relationship between the missile and the maneuvering target like the above assumptions cannot be predicted, so it is difficult to set a suitable desired LOS angle in advance.

Motivated by the aforementioned papers, in order to achieve the dynamic surrounding attack of multiple missiles against a highly maneuvering target and further consider the couplings between the LOS and normal LOS directions in the cooperative guidance model, we proposed the ITDSG in this paper. The main contributions of this paper can be summarized as follows:

(1) We proposed the strategy of dynamic surrounding attack and established the cooperative guidance model between multiple missiles and virtual targets. Due to the traditional cooperative guidance laws with angle constrain need a preset LOS angle [26-29], it is difficult to set the appropriate angle in advance due to the unknown maneuver of the target. But the dynamic surrounding attack strategy proposed in this paper can avoid this

(2) By using the dynamic inverse method, the coupled cooperative guidance model is decoupled, and the ITDSG is designed based a prescribed-time stable method subject to the decoupled model. This is opposed to Ref $[21,22,30]$, where the guidance laws are designed without considered the coupling
(3) The proposed cooperative guidance in this paper requires less maximum overload and energy consumption compared with Ref [21], which is more conducive to engineering realization

The remainder of this paper is organized as follows. Section 2 presents some necessary preliminaries and model description. Section 3 presents the dynamic surrounding attack strategy and the detailed design processes of proposed cooperative guidance law. Finally, the effectiveness of dynamic surrounding attack strategy and cooperative guidance law are verified through simulations in Section 4; conclusions are drawn in Section 5.

\section{Problem Formulation}

2.1. Model Description. In this section, the model description and the basic knowledge of consensus protocol are introduced.

The relative motion geometry of single missile and single target can be formulated as follows:

$$
\begin{gathered}
\dot{r}=V_{t} \cos \left(q-\theta_{t}\right)-V_{m} \cos \left(q-\theta_{m}\right), \\
r \dot{q}=-V_{t} \sin \left(q-\theta_{t}\right)+V_{m} \sin \left(q-\theta_{m}\right) .
\end{gathered}
$$

Differentiating Eqs. (1) and (2) with respect to time $t$, the equations are obtained as follows:

$$
\begin{array}{r}
\ddot{r}=w_{r}-u_{r}+r \dot{q}^{2}, \\
\ddot{q}=\frac{w_{q}-2 u_{q}-2 \dot{r} \dot{q}}{r} .
\end{array}
$$

In Eqs. (3) and (4), $u_{r}$ and $w_{r}$ denote the components of the acceleration of the missile and target in the LOS direction, respectively; $u_{q}$ and $w_{q}$ denote components of the acceleration of the missile and target in the normal LOS direction, respectively.

In order to ensure that missiles hit the target simultaneously, a variable $t_{\mathrm{go}}$ called time-to-go is introduced in this paper, which can be estimated as

$$
t_{g o}=-\frac{r}{\dot{r}}
$$

Compute the derivation of $t_{g o}$ with respect time $t$ as follows:

$$
\dot{t}_{g o}=-1+\frac{r^{2} \dot{q}^{2}}{\dot{r}^{2}}-\frac{r}{\dot{r}^{2}} u_{r}+\frac{r}{\dot{r}^{2}} w_{r}
$$

Combining Eqs. (1) and (2) with Eqs. (4) and (6), the state equations of the $i$ th missile can be described as follows: 


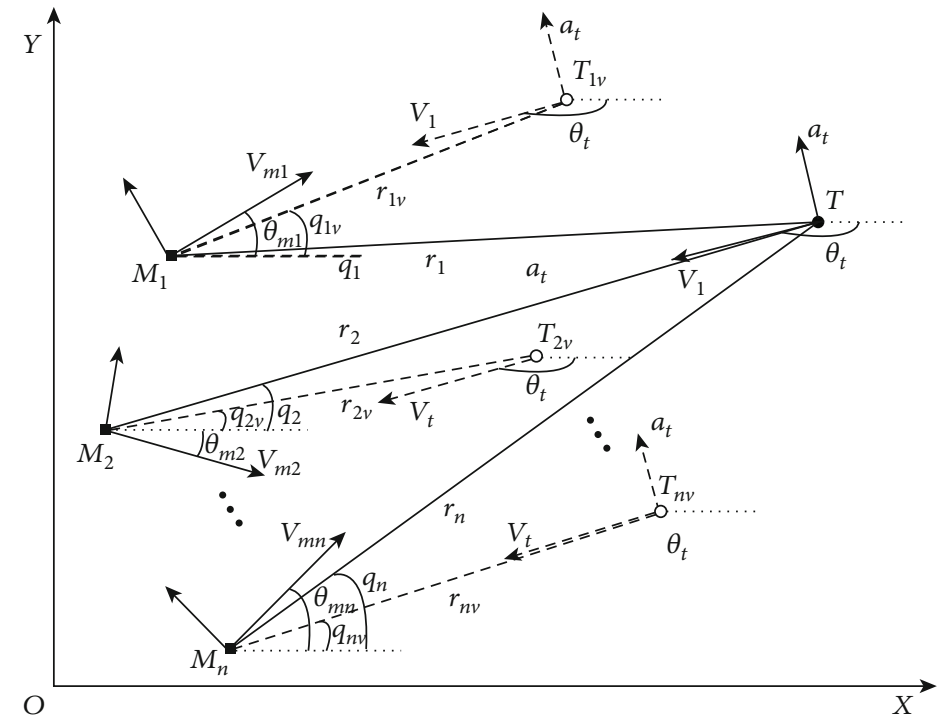

FIGURE 1: Guidance geometry on Missiles-Target (virtual targets) engagement.

$$
\left\{\begin{array}{l}
\dot{x}_{1 i}=\frac{r_{i}^{2}}{\dot{r}_{i}^{2}} x_{2 i}^{2}-\dot{T}_{f i}^{*}-\frac{r_{i}}{\dot{r}_{i}^{2}} u_{r i}+d_{r i}, \\
\dot{x}_{2 i}=-\frac{2 \dot{r}_{i}}{r_{i}} x_{2 i}-\frac{1}{r_{i}} u_{q i}+d_{q i},
\end{array}\right.
$$

where $x_{1 i}=t_{g o i}+t-T_{f i}^{*}$ and $x_{2 i}=\dot{q}_{i}$ are the state variables and $d_{r i}=\left(r / \dot{r}^{2}\right) w_{r}$ and $d_{q i}=\left(w_{q} / r\right)$ are the disturbances caused by target maneuver.

Considering the problem of $n$ missiles intercept a maneuvering target simultaneously, a dynamic surrounding attack strategy is proposed in this paper. By this new strategy, the problem is transformed to that multiple missiles attack multiple virtual, where the virtual targets approach the real target with missiles approaching the real target. Figure 1 shows the guidance geometry of Missiles-Target-Virtual Targets in two-dimensional plane, where $M_{i}$ and $T_{i v} i=(1,2, \cdots, n)$ represent the $i$ th missile and the $i$ th virtual target; $T$ denotes the real target; $V_{m i}$ and $\theta_{m i} i=(1,2, \cdots, n)$ denote the velocity and the flight path angle of $i$ th missile respectively; $a T$ is the normal acceleration of target; $V t$ and $\theta t$ denote the velocity and the flight path angle of target, respectively; $a T$ is the normal acceleration of target; and $q_{i}$ and $r_{i} i=(1,2, \cdots, n)$ denote the LOS angle and distance between $i$ th missile and $i$ th virtual target, respectively. The subscripts $m, t$, and $v$ denote the state variables of missiles, target, and virtual targets, respectively. For multimissiles against single target system, the subscript $i$ denotes the ith missile's or virtual target's state variable.

Assumption 1. The disturbance $d_{r i}$ and $d_{q i}$ are bounded, that is $\left|d_{r i}\right|<\varepsilon_{r i}$ and $\left|d_{q i}\right|<\varepsilon_{q i}$, where $\varepsilon_{r i}$ and $\varepsilon_{q i}$ are the given positive constants.

2.2. Graph. Suppose that communication network graph between agents can be expressed as $G=\{V, E, C\}$, where $V$ $=\{1,2, \cdots, n\}$ denotes the set of vertices, $E \subseteq V \times V$ denotes the edge set of graph, the subscripts $i$ denotes the $i$ th agent, $e_{i j}$ is the edge of graph $G$, and $e_{i j} \subseteq E$ indicates that the agent $i$ and $j$ can receive message from each other in an undirected graph. If there is a connection between any two agents in the graph, then the graph $G$ is connected. In the directed graph, $e_{i j} \subseteq E$ means that the agent $i$ can receive message from agent $j$. In addition, $C=\left(c_{i j}\right) \in R^{n \times n}$ is the adjacency matrix of graph $G$. If $e_{i j} \subseteq E$, then $c_{i j}>0$; otherwise, $c_{i j}=0$. It is worth noting that $c_{i j}=c_{j i}$ when the communication topology is an undirected graph. The Laplace matrix of the graph $G$ is defined as $L=\left(L_{i j}\right) \in R^{n \times n}$, which can be expressed as:

$$
L_{i j}= \begin{cases}-c_{i j} & i \neq j, \\ \sum_{j=1, j \neq i}^{n} c_{i j} & i=j .\end{cases}
$$

In this paper, the communication network graph of multiple missiles is assumed to be undirected and connected.

2.3. Multiagent Consensus. The first-order multiagent system with $n$ agents is described as

$$
\dot{x}_{i}=u_{i}
$$

where $x_{i}$ denotes the state of $i$ th agent and $u_{i}$ is the consensus protocol to be designed with the information of the $i$ th agent and its neighbors.

Lemma 2 [31]. For a first-order multiagent system as Eq. (9), if the graph of communication topology is undirected and connected, the state variables $x_{i}(i=1, \cdots, N)$ of $n$ agents can be convergent in a prescribed-time $T$ by the following consensus protocol.

$$
u_{i}=-\left(k+c \frac{\dot{\mu}(t)}{\mu(t)}\right) \sum_{j \in N_{i}} a_{i j}\left(x_{i}-x_{j}\right)
$$




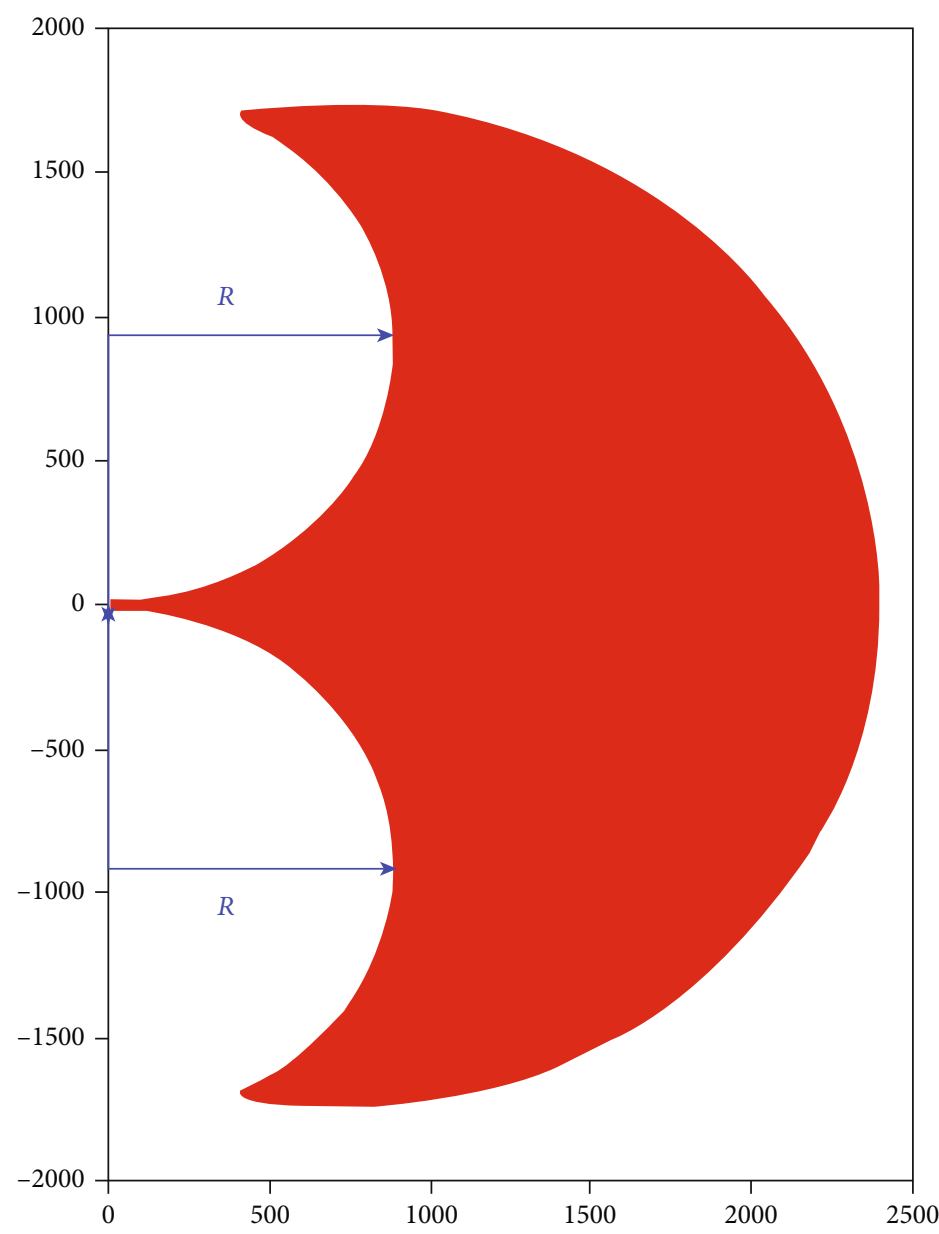

(a) Target escape area in Case

Figure 2: Continued. 


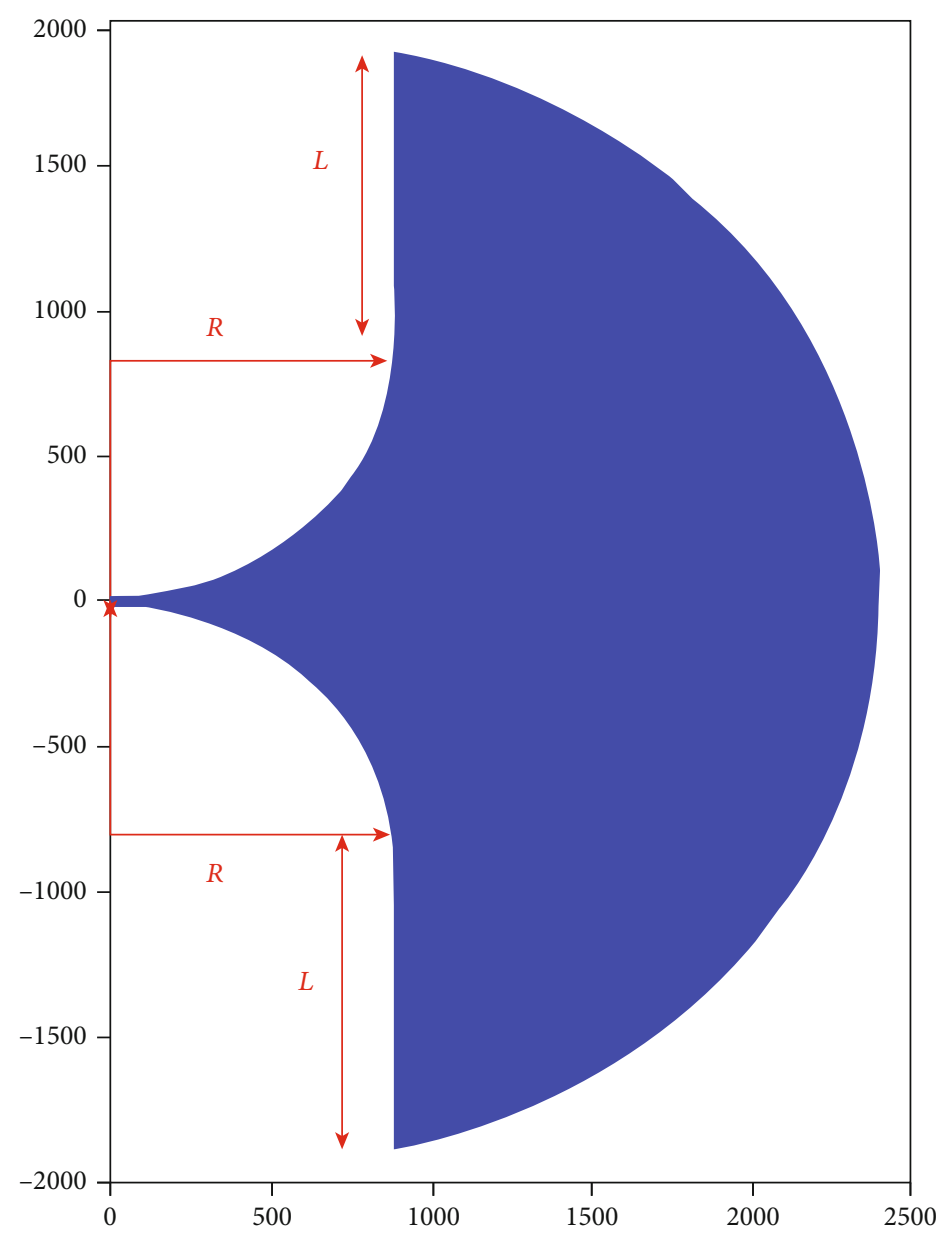

(b) Target escape area in Case 2

Figure 2: Target escape area.

where $i=1, \cdots, N ; k>0$ and $c>1$ are design parameters; and $\mu(t)$ is a time-varying scaling function as

$$
\mu(t)=\left\{\begin{array}{ll}
\frac{T^{h}}{\left(T+t_{0}-t\right)^{h}}, & t \in\left[t_{0}, T\right), \\
1, & t \in[T, \infty)
\end{array},\right.
$$

where $h>2$ is a real number and $T>T_{s}>0$ with the minimum communication interval $T_{s}$.

Lemma 3 [32]. Consider a nonlinear system defined as

$$
\dot{x}(t)=f(t, x(t))+d(t)+u, t \in R_{+} .
$$

Select a continue and differentiable Lyapunov candidate function $V(t, x(t))>0$ and $V(t, 0)=0$. The state vector $x$ is prescribed-time stable with a given time $T$ in Eq. (11), if the differentiation of Lyapunov function satisfies $\dot{V} \leq-b V-k(\dot{\mu}$ $/ \mu)$, on $t \in\left[t_{0}, \infty\right)$. In addition, the Lyapunov candidate function holds

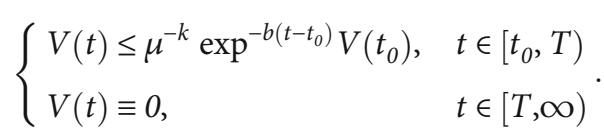

Lemma $4[16,33]$. For a nonlinear system with an unknown and bounded disturbance $d(t)$ as Eq. (12). Suppose that the state vector $x$ and the control input $u$ can be measured. By a second order ESO Eq. (14), there exist observer gain $\gamma_{11}$, $\gamma_{12}, \mu_{1}$, and $\delta_{1}$ such that the estimated states $Z_{11}$ and $Z_{12}$ converge into a neighborhood actual states $x$ and $d(t)$, respectively.

$$
\left\{\begin{array}{l}
E_{11}=Z_{11}-x \\
\dot{Z}_{11}=Z_{12}+f(t, x(t))+u-\gamma_{11} E_{11} \\
\dot{Z}_{12}=-\gamma_{12} f a l\left(E_{11}, \mu_{1}, \delta_{1}\right)
\end{array}\right.
$$

where $E_{11}$ is the observed error of the state $x ; \gamma_{11}, \gamma_{12}, \mu_{1}$, and $\delta_{1}$ are the parameters of ESO; and fal is defined as 


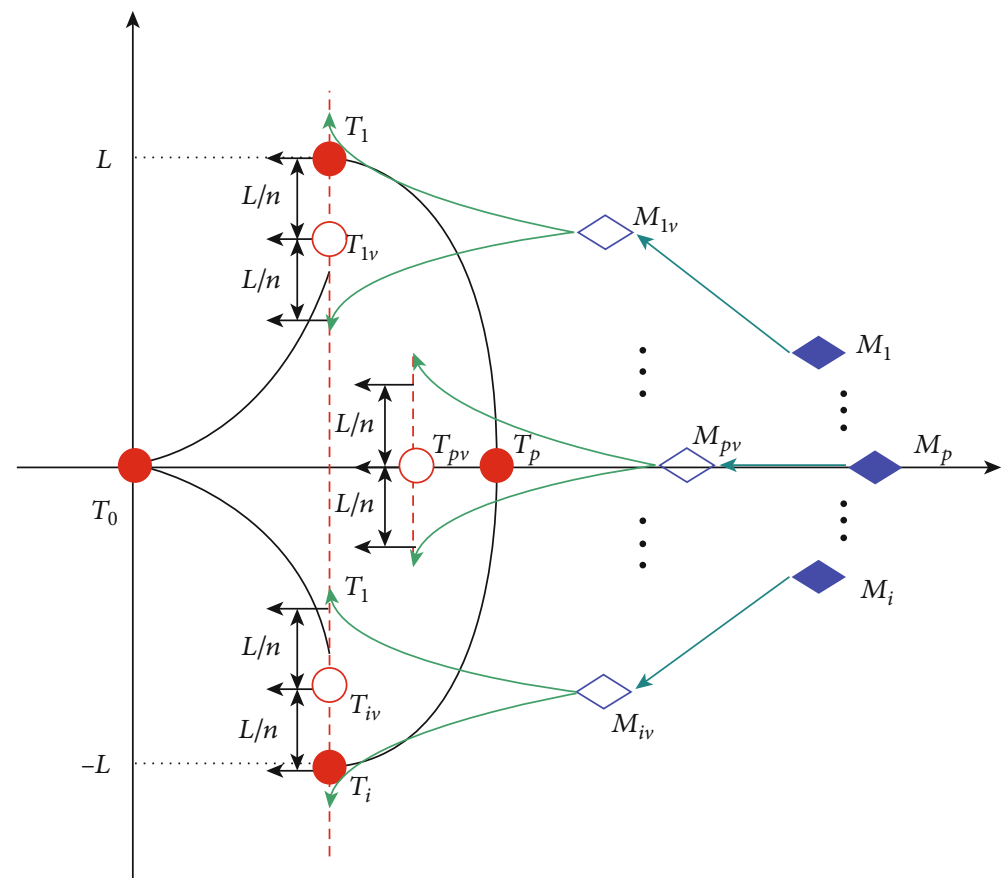

FIgURE 3: Guidance geometry on Missiles-Targets engagement.

$$
f a l\left(E_{11}, \mu_{1}, \delta_{1}\right)=\left\{\begin{array}{ll}
\left|E_{11 i}\right|^{\mu_{1}} \operatorname{sgn}\left(E_{11}\right), & \left|E_{11}\right|>\delta_{1} \\
E_{11} / \delta_{1}{ }^{1-\mu_{1}}, & \left|E_{11}\right| \leq \delta_{1}
\end{array} .\right.
$$

\section{Main Result}

Firstly, a novel dynamic surrounding attack strategy is proposed in this section by introducing the virtual targets. Then, we propose the calculation formulas of the position of virtual targets using measurable real target information. Finally, considering the couplings between the LOS and normal LOS directions of the guidance model in Eq. (7), an adaptive dynamic inverse method is proposed to decouple them. And an impact time and dynamic surrounding cooperative guidance law (ITDSG) is designed subject to the decoupled models. Besides, the accelerations of target in LOS and normal LOS directions are regarded as disturbances and estimated by ESOs.

Remark 5. The difference between dynamic and static surrounding attack is whether a preset LOS angle constraint is required. The static surrounding attack is achieved by presetting different LOS angle constraint for each missile to achieve multiple missiles attacking the target from different directions. This attack strategy is determined at the beginning of the missile terminal guidance, which will not change with the maneuvering of the target. By introducing multiple virtual targets, the dynamic surrounding attack strategy without preset LOS angle constraint is achieved. The virtual targets are calculated according to the movement of real target, and multiple virtual targets gradually approach the real target from different directions.
3.1. The Strategy of Dynamic Surrounding Attacking. In the scenario of single missile intercepting a target, at the terminal phase of intercepting, the target usually maneuvers to avoid being hit by a missile. Figure 2(a) shows the target escape area, where target moves with a fixed overload from $n_{T \max }$ to $n_{T \max }$ in a finite time. $R$ denotes the minimum turning radius of the target. In a real combat environment, the purpose of the target turning left or right is to obtain a maximum lateral distance perpendicular to the line of sight. When the target is maneuvering with the abovementioned strategy, escape area of the target is shown in Figure 2(b). $R$ denotes the minimum turning radius of the target; $L$ denotes the distance of the target flying along a straight line after a $90^{\circ}$ turning.

Figure 3 shows the novel guidance strategy ITDSG. In the scenario that $n$ missiles intercept a maneuvering target, where $T_{0}$ and $M_{i}(i=1,2, \cdots, n)$ represent the initial positions of target and missiles, respectively. $T_{1}$ represents the upper boundary point of the target perpendicular to the initial velocity direction in the forward maximum positive overload maneuver. Similarly, $T_{i}$ represents the lower boundary point when the target maneuvers with negative maximum overload. $T_{p}$ is the middle boundary point of the target with a zero overload. The escape area is determined by the velocity and the maximum overload of the target. In this paper, the escape area of the target is divided into $n$ subescape areas, where $n$ is the number of missiles; the center point of the subescape area is set as the virtual target which denotes as $T_{i v}(i=1,2, \cdots, n)$. The goal of cooperative guidance is to make the reachable sets of the $n$ missiles cover these $n$ subescape areas, respectively. When the distances between missiles and the virtual targets become smaller, their times-to-go gradually approach zero, and multiple virtual targets gradually approach the real target from different 
directions as well as the escape areas of multiple virtual targets coincide gradually. Since each missile aiming at a virtual target, thus the dynamic surrounding attack is achieved.

3.2. Dynamic Models of Missiles and Virtual Targets. The dynamic models of the missiles and virtual targets can be established by the three steps. Firstly, calculate the coordinate of boundary points subject to the real target position and velocity information. Then, the coordinates of $n$ virtual targets are calculated based on the information of the boundary points. Finally, by using the information of virtual targets and the existing dynamic model of single missile and target, the dynamic models of multiple missiles and multiple virtual targets are established.

Step 1. Calculate the boundary point.

Figure 4 shows the geometry between the target boundary point with the target initial position and velocity. The time of the target flying a quarter of a circle with maximum overload is defined as $T_{q c}$. $T_{0}$ denotes the initial point of the target; $T_{u 1}$ and $T_{u 2}$ represent the lateral boundary point when the target is maneuvering with a positive overload and the flight time is less and greater than $T_{q c}$, respectively; $T_{d 1}$ and $T_{d 2}$ represent the lateral boundary point when the target is maneuvering with a negative overload and the flight time is less and greater than $T_{q c}$, respectively; $T_{g}$ represents the boundary point when the target is moving in a straight line without any maneuvering.

However, the seeker can only obtain the relative information of the missile and the target, such as $r, q, \dot{r}$, and $\dot{q}$; the information of the missile such as the position $\left(x_{m i}, y_{m i}\right)$ and velocity $\left(V_{m i}, \theta_{m i}\right)$ can be obtained through its own inertial navigation device; the velocity of the target $\left(V_{t}\right.$, $\theta_{T}$ ) can be calculated with Eqs. (1) and (2); the target's coordinates can be calculated as $x_{T}=x_{m i}+r \cos \left(q_{i}\right)$ and $y_{T}=y_{m i}+r \sin \left(q_{i}\right)$. Suppose that at least one missile can obtain the position and velocity of the target which are transmitted to other missiles through the communication network. Then, each missile can calculate boundary points and virtual target points based on the real target information.

The boundary points can be calculated as follows. When $T_{p} \leq T_{q c}$, then

$$
\begin{aligned}
& \left\{\begin{array}{l}
x_{u 1}=-R_{T}[1-\cos (\alpha)] \sin \left(\theta_{t}\right)+R_{T} \sin (\alpha) \cos \left(\theta_{t}\right)+x_{T 0}, \\
y_{u 1}=R_{T}[1-\cos (\alpha)] \cos \left(\theta_{t}\right)+R_{T} \sin (\alpha) \sin \left(\theta_{t}\right)+y_{T 0},
\end{array}\right. \\
& \left\{\begin{array}{l}
x_{d 1}=R_{T}[1-\cos (\alpha)] \sin \left(\theta_{t}\right)+R_{T} \sin (\alpha) \cos \left(\theta_{t}\right)+x_{T 0}, \\
y_{d 1}=-R_{T}[1-\cos (\alpha)] \cos \left(\theta_{t}\right)+R_{T} \sin (\alpha) \sin \left(\theta_{t}\right)+y_{T 0},
\end{array}\right.
\end{aligned}
$$

when $T_{p}>T_{q c}$, then

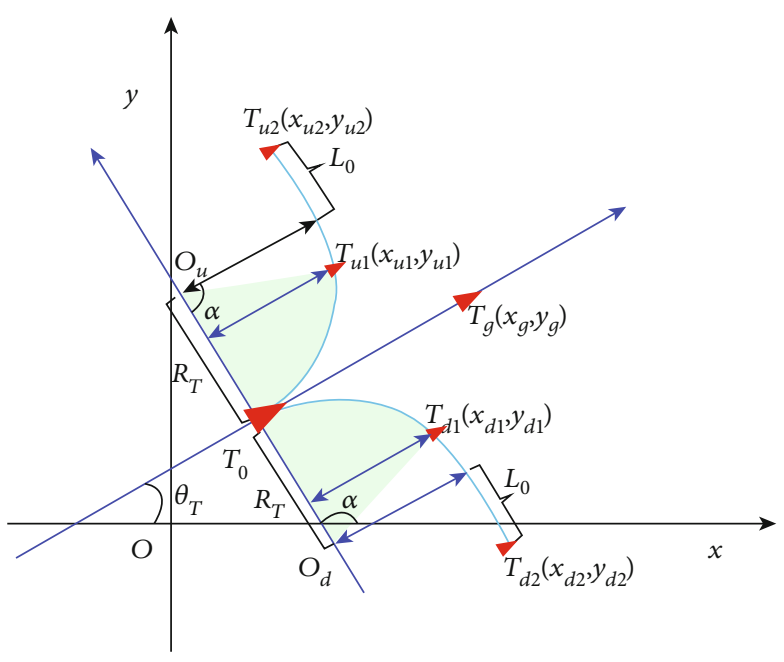

FIgURE 4: Relative geometrical between target boundary point and initial position of target.

$$
\begin{aligned}
& \left\{\begin{array}{l}
x_{u 2}=-\left(R_{T}+L_{0}\right) \sin \left(\theta_{t}\right)+R_{T} \cos \left(\theta_{t}\right)+x_{T 0}, \\
y_{u 2}=\left(R_{T}+L_{0}\right) \cos \left(\theta_{t}\right)+R_{T} \sin \left(\theta_{t}\right)+y_{T 0},
\end{array}\right. \\
& \left\{\begin{array}{l}
x_{d 2}=\left(R_{T}+L_{0}\right) \sin \left(\theta_{t}\right)+R_{T} \cos \left(\theta_{t}\right)+x_{T 0}, \\
y_{d 2}=-\left(R_{T}+L_{0}\right) \cos \left(\theta_{t}\right)+R_{T} \sin \left(\theta_{t}\right)+y_{T 0} .
\end{array}\right.
\end{aligned}
$$

What is more, the coordinates $\left(x_{g}, y_{g}\right)$ of the bounded point can be calculated as:

$$
\left\{\begin{array}{l}
x_{g}=V_{t} T_{p} \cos \left(\theta_{t}\right)+x_{T 0} \\
y_{g}=V_{t} T_{p} \sin \left(\theta_{t}\right)+y_{T 0}
\end{array}\right.
$$

where $R_{T}=V t^{2} / a_{t \text { max }}$ denotes the minimum turning radius of the target, $\theta_{t}$ is the flight path angle of the target, $T_{0}$ is the initial position of the target denoted as $\left(x_{T 0}, y_{T 0}\right)$, and the other variables can be calculated as $T_{q c}=\pi R_{T} / 2 V_{T}, \alpha=$ $\pi T_{p} / 2 T_{q c}$, and $L_{0}=V_{T}\left(T_{p}-T_{q c}\right)$.

Step 2. Calculate the coordinates of virtual targets.

Considering the scenario that $n$ missiles cooperatively intercept a highly maneuvering target, based on the ITDSG interception strategy, the missile's attack range covers the escape area of the target by introducing $n$ virtual targets. The coordinates $\left(x_{i v}, y_{i v}\right)$ of the virtual targets $T_{i v}(i=1$, $2, \cdots, n)$ can be calculated as follows. When $T_{p}>T_{q c}$, then

$$
\left\{\begin{array}{l}
x_{i v}=-\frac{n-2 i+1}{n}\left(R_{T}+L_{0}\right) \sin \left(\theta_{t}\right)+R_{T} \cos \left(\theta_{t}\right)+x_{T 0}, \\
y_{i v}=\frac{n-2 i+1}{n}\left(R_{T}+L_{0}\right) \cos \left(\theta_{t}\right)+R_{T} \sin \left(\theta_{t}\right)+y_{T 0} .
\end{array}\right.
$$


When $T_{p} \leq T_{q c}$, then

$\left\{\begin{array}{l}x_{i v}=-\frac{n-2 i+1}{n} R_{T}[1-\cos (\alpha)] \sin \left(\theta_{t}\right)+R_{T} \sin (\alpha) \cos \left(\theta_{t}\right)+x_{T 0}, \\ y_{i v}=\frac{n-2 i+1}{n} R_{T}[1-\cos (\alpha)] \cos \left(\theta_{t}\right)+R_{T} \sin (\alpha) \sin \left(\theta_{t}\right)+y_{T 0},\end{array}\right.$

where $(n-2 i+1) / n(i=1,2, \cdots, n)$ is designed to adjust the position of $i$ th virtual target in the normal direction of target velocity. By setting the virtual targets, the reachable sets of all missiles can cover the escape area of the target. In Eqs. (19) and (20), $T_{p}$ determines the values $R_{T}$ and $L_{0}$, which is defined as follows:

$$
T_{p}(t)= \begin{cases}T_{p \max }, & t<T_{s} \\ T_{g o p}-t-\delta t, & T_{s} \leq t \leq T_{e} \\ 0, & t>T_{e}\end{cases}
$$

where $T_{s}=T_{g o p}-\delta t-T_{p \max }, T_{e}=T_{\text {gop }}-\delta t, T_{\text {gop }}=T_{\text {go }}(0)$, $T_{p \max }$, and $\delta_{t}$ are the given positive constants and $t$ represents the flight time.

As a result, by applying Eqs. (19)-(21), the position of virtual targets $\left(x_{i v}, y_{i v}\right)(i=1,2, \cdots, n)$ can be obtained.

Step 3. Establish the dynamic model.

Similar to Eq. (7), the dynamic models of multiple missiles attacking multiple virtual targets based on the information of the virtual targets can be written as follow

$$
\left\{\begin{array}{l}
\dot{x}_{1 i v}=\frac{r_{i v}^{2}}{\dot{r}_{i v}^{2}} x_{2 i v}^{2}-\dot{T}_{f i v}^{*}-\frac{r_{i v}}{\dot{r}_{i v}^{2}} u_{r i}+d_{r i v} \\
\dot{x}_{2 i v}=-\frac{2 \dot{r}_{i v}}{r_{i v}} x_{2 i v}-\frac{1}{r_{i v}} u_{q i}+d_{q i v}
\end{array}\right.
$$

where $\quad x_{1 i v}=t_{\text {goiv }}+t-T_{f i v}=-\left(r_{i v} / \dot{r}_{i v}\right)+t-T_{f i v} ; \quad x_{2 i v}=\dot{q}_{i v}$; $r_{i v}=\sqrt{\left(x_{i v}-x_{m i}\right)^{2}+\left(y_{i v}-y_{m i}\right)^{2}} ; \quad \dot{q}_{i v}=d q / d t ; \quad \dot{r}_{i v}=d r / d t$ $; q_{i v}=\arctan \left(y_{i v}-y_{m i}\right) /\left(x_{i v}-x_{m i}\right) ; x_{m i}$ and $y_{m i}$ are the coordinates of missile $i$, which are obtained from the missile's inertial navigation system, $x_{i v}$ and $y_{i v}$ cannot be measured, which can be calculated by Step 1 and Step 2. Besides, $d_{\text {riv }}$ $=\left(r_{i v} / \dot{r}_{i}^{2}\right) w_{r}+d_{\text {rie }}$ and $d_{q i v}=\left(1 / r_{i v}\right) w_{q}+d_{q i \epsilon}$ are the disturbances, where $w_{r}$ and $w_{q}$ are the components of the real target's acceleration in LOS and norm LOS directions and $d_{\text {rie }}$ and $d_{\text {qie }}$ are the virtual acceleration caused by the virtual target approaching the real target.

In the initial stage of intercepting, the $i$ th missile is aiming at the $i$ th virtual target. Then, with the time $t$ tending to $T_{e}$, the virtual targets are approaching the real target. In the final stage, the virtual targets and real target are coincident, that is, all missiles aim at the real target.

3.3. The Guidance Law Design of ITDSG. In this section, an adaptive dynamic inverse method is used to decouple and
TABLE 1: Simulation conditions for missiles.

\begin{tabular}{lccccc}
\hline Missile & $\begin{array}{c}\text { Initial } \\
\text { position }(\mathrm{m})\end{array}$ & $\theta m i 0\left(^{\circ}\right)$ & $a_{m i}(g)$ & $V_{m i 0}(\mathrm{~m} / \mathrm{s})$ & $R_{0}(\mathrm{~km})$ \\
\hline Missile 1 & $(8000,0)$ & 2 & $\pm 30 \mathrm{~g}$ & 600 & 18 \\
Missile 2 & $(9000,1500)$ & 6 & $\pm 30 \mathrm{~g}$ & 600 & 19 \\
Missile 3 & $(7000,-800)$ & -6 & $\pm 30 \mathrm{~g}$ & 600 & 17 \\
\hline
\end{tabular}

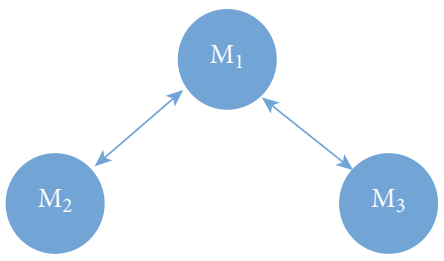

Figure 5: Communication topology for three missiles.

linearize Eq. (22); then, the ITDSG is designed with a prescribed-time stable control method. Equation (22) can be rewritten as

$$
\dot{\mathbf{x}}_{i}=\mathbf{f}_{i}\left(\mathbf{x}_{i}\right)+\mathbf{g}_{i}\left(\mathbf{x}_{\mathbf{i}}\right) \mathbf{u}_{i}+\mathbf{d}_{i}(t)
$$

where

$$
\begin{aligned}
\mathbf{f}_{i}\left(\mathbf{x}_{i}\right)= & {\left[\begin{array}{c}
\frac{r_{i v}^{2}}{\dot{r}_{i v}{ }^{2}} x_{3 i v}^{2} \\
-\frac{2 \dot{r}_{i v}}{r_{i v}} x_{3 i v}
\end{array}\right], \mathbf{u}_{i}=\left[\begin{array}{l}
u_{r i} \\
u_{q i}
\end{array}\right], } \\
\mathbf{g}_{i}\left(x_{i}\right)= & {\left[\begin{array}{cc}
-\frac{r_{i v}}{\dot{r}_{i v}{ }^{2}} & 0 \\
0 & -\frac{1}{r_{i} v}
\end{array}\right], \mathbf{d}_{i}(t)=\left[\begin{array}{l}
d_{r i v} \\
d_{q i v}
\end{array}\right] . }
\end{aligned}
$$

Applying Lemma 4, the disturbances $d_{\text {riv }}$ and $d_{q i v}$ can be estimated by $\widehat{d}_{\text {riv }}$ and $\widehat{d}_{\text {qiv }}$ with two ESOs, and there exist bounded real numbers $\eta_{1 i}>0$ and $\eta_{2 i}>0$ satisfying $\mid d_{\text {riv }}-$ $\widehat{d}_{\text {riv }}\left|\leq \eta_{1 i},\right| d_{\text {qiv }}-\widehat{d}_{\text {qiv }} \mid \leq \eta_{2 i}$. The inverse matrix of $\mathbf{g}_{i}\left(\mathbf{x}_{i}\right)$ can be calculated as follows:

$$
\mathbf{g}_{i}\left(\mathbf{x}_{i}\right)^{-1}=\left[\begin{array}{cc}
-\frac{\dot{r}_{i}^{2}}{r_{i}} & 0 \\
0 & -r_{i}
\end{array}\right]
$$

According to the dynamic inverse method, the controller can be computed as follows:

$$
\mathbf{u}_{i}(t)=\mathbf{g}_{i}\left(\mathbf{x}_{i}\right)^{-1}\left[\mathbf{V}_{i}(t)-\mathbf{f}_{i}\left(\mathbf{x}_{i}\right)-\mathbf{d}_{i}(t)\right]
$$

Substituting the above controller $\mathbf{u}_{i}(t)$ into Eq. (23), we obtain

$$
\dot{\mathbf{x}}_{i}(t)=\mathbf{V}_{i}(t)
$$




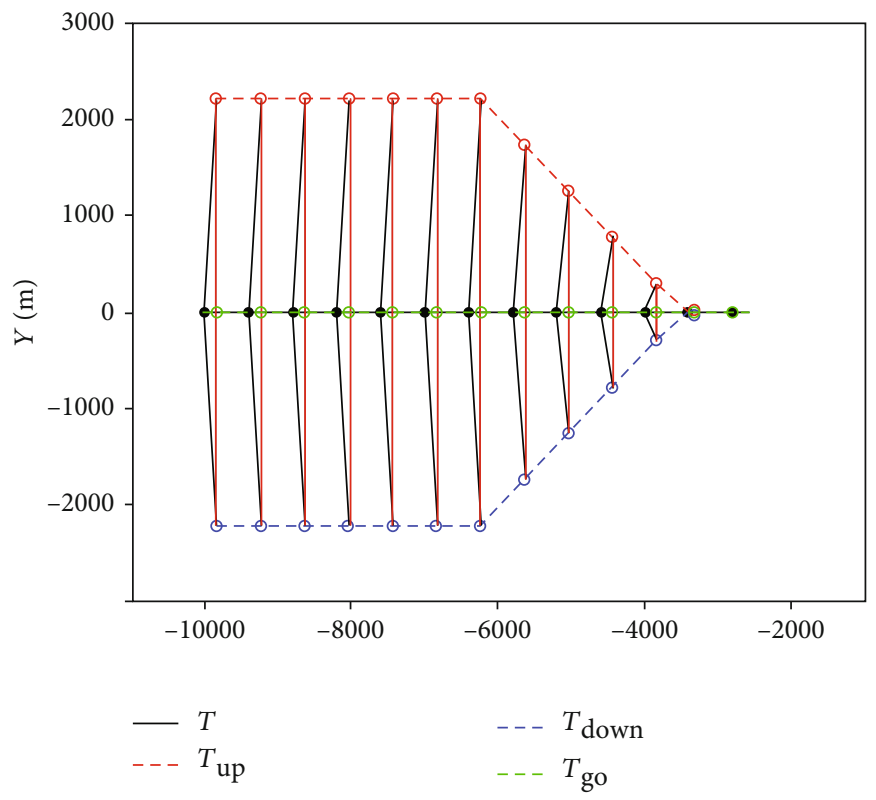

Figure 6: Virtual targets with constant velocity target.

The above equation is the expected linearization one; $\mathbf{V}_{i}(t)$ is the desired dynamic equation of $x_{i}$, considering the estimation errors of the disturbances and applying prescribed-time control method; the desired dynamic equation of $\mathbf{x}_{i}$ can be designed as:

$$
\mathbf{V}_{i}(t)=-\mathbf{K}_{i}(t) \mathbf{x}_{i},
$$

where

$\mathbf{K}_{i}(t)=\operatorname{diag}\left[k_{11 i}+k_{12 i} \frac{\mu(t)}{\mu(t)}+\eta_{1 i} \operatorname{sign}\left(x_{1 i v}\right), k_{21 i}+k_{22 i} \frac{\mu(t)}{\mu(t)}+\eta_{2 i} \operatorname{sign}\left(x_{1 i v}\right)\right]$,

is a diagonal matrix to be designed, where $k_{11 i}>0, k_{12 i}>0$, $k_{21 i}>0, k_{22 i}>0, \eta_{1 i}$, and $\eta_{2 i}$ are the parameters of controller.

Finally, combining with the Eqs. (26)-(28), the controller is designed as Eq. (30).

Based on the above design method of the decoupled guidance model, a nonlinear and strong coupled guidance problem can be transformed into linear control stabilization problem. By designing the controller, all state variables can converge to $0 . x_{1 i v}=0$ means $t_{g o f i}(i=1,2, \cdots, n)$ of all $n$ missiles tend to a same value. When $x_{2 i v}=0$ is satisfied, each missile can be guaranteed to hit the target.

$$
\left[\begin{array}{c}
u_{r i} \\
u_{q i}
\end{array}\right]=\left[\begin{array}{c}
\frac{\dot{r}_{i v}{ }^{2}}{r_{i v}}\left[-\left(k_{11 i}+k_{12 i} \frac{\mu(t)}{\mu(t)}+\eta_{1 i} \operatorname{sign}\left(x_{1 i v}\right) x_{1 i v}-\frac{r_{i v}{ }^{2}}{\dot{r}_{i v}} x_{2 i v}^{2}+u_{i}^{\text {nom }}-\widehat{d}_{r i v}\right]\right. \\
\left.-r_{i v}\left(k_{21 i}+k_{22 i} \frac{\mu(t)}{\mu(t)}+\eta_{2 i} \operatorname{sign}\left(x_{2 i v}\right)\right)\right)-2 \dot{r}_{i} x_{2 i v}-\hat{d}_{q i}
\end{array}\right] .
$$

Remark 6. It is easy to obtain that, as $t$ tends to $T, \mu(t)$ and $\dot{\mu}(t) / \mu(t)$ both approach to infinity, which may cause the control input to be infinity. However, the state values $x_{1 i}$ and $x_{2 i}$ will approach zero at the prescribed-time $T$, such that the control input $u_{r i}$ and $u_{q i}$ are both bounded, which has been proved in Ref. [31]. In addition, due to the use of a prescribed-time control method, the convergence rate of the state variables is slower in the initial phase, when the time $t$ approaches $T$, the convergence rate gradually increases. This will avoid large control inputs in the initial stage, which is more conducive to engineering realization.

Theorem 7. For the multiple-missile and multiple-virtual target system Eq. (23), suppose that the disturbances $d_{r i}$ and $d_{q i}$ can be estimated by ESOs designed as Eq. (14). The proposed guidance Eq. (30) can guarantee that all the $t_{\text {gofi }}$ converge to a same value (all the missiles hit the target simultaneously), and $q^{\cdot}{ }_{i}$ converges to 0 (each missile can hit the target) in a prescribed-time $T$, respectively.

Proof. Define the following Lyapunov candidate function:

$$
\mathbf{V}_{1}=\frac{1}{2} \mathbf{x}_{i}^{T} \mathbf{x}_{i}
$$

The derivative of Eq. (31) is given as

$$
\dot{\mathbf{V}}_{1}=\mathbf{x}_{i}{ }^{T} \dot{\mathbf{x}}_{i}
$$

By applying Eqs. (30) and (23) to Eq. (32) and rearranging, the following inequality can be obtained: 


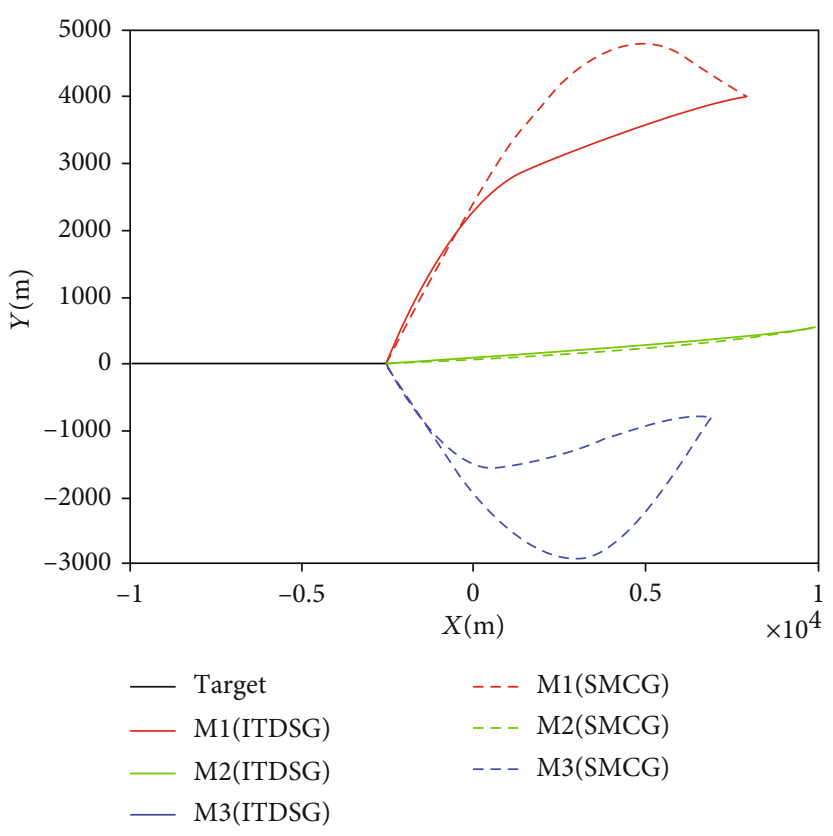

(a) Trajectories for missiles and target

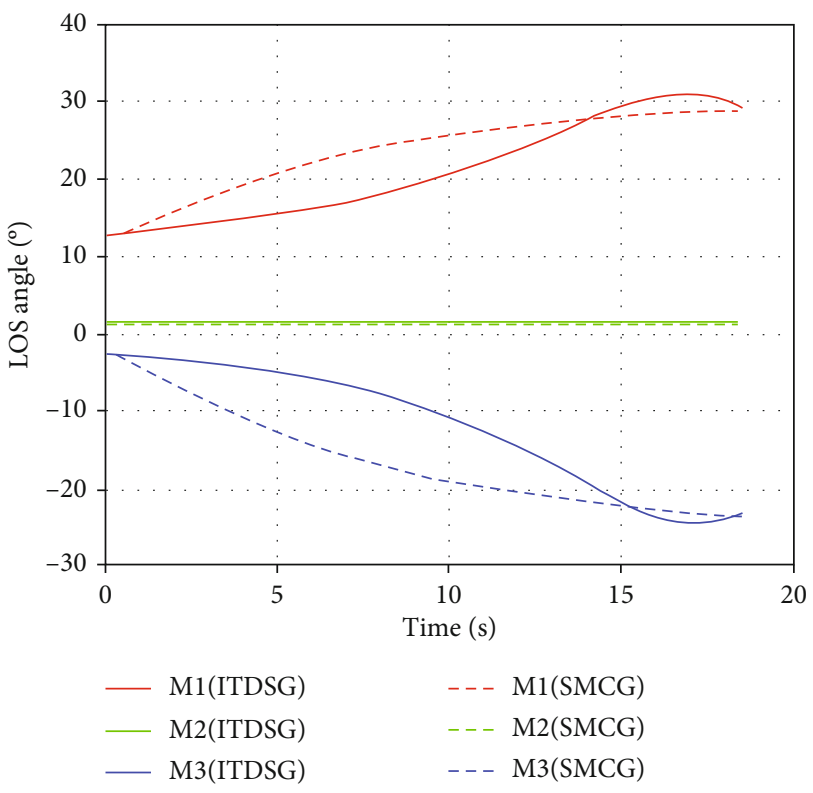

(c) LOS angles for missiles

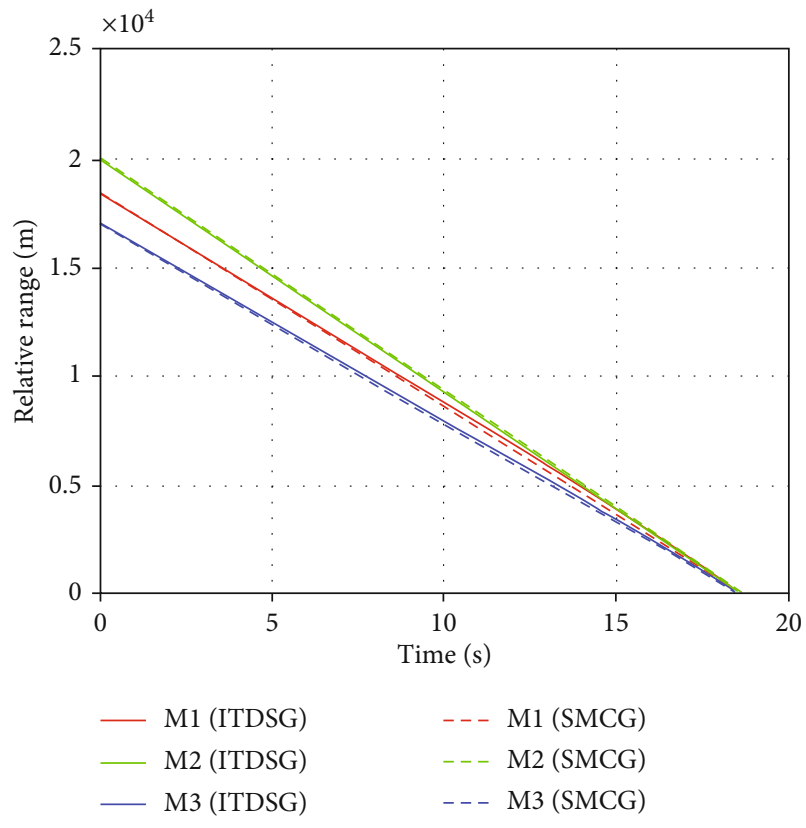

(b) Distance between missiles and target

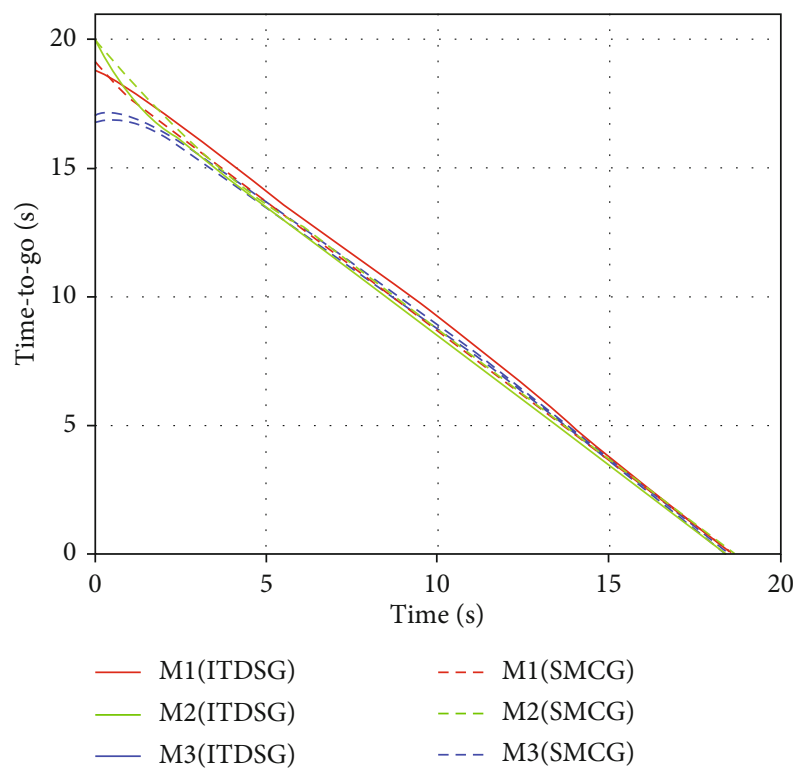

(d) Time-to-go for missiles

Figure 7: Continued. 


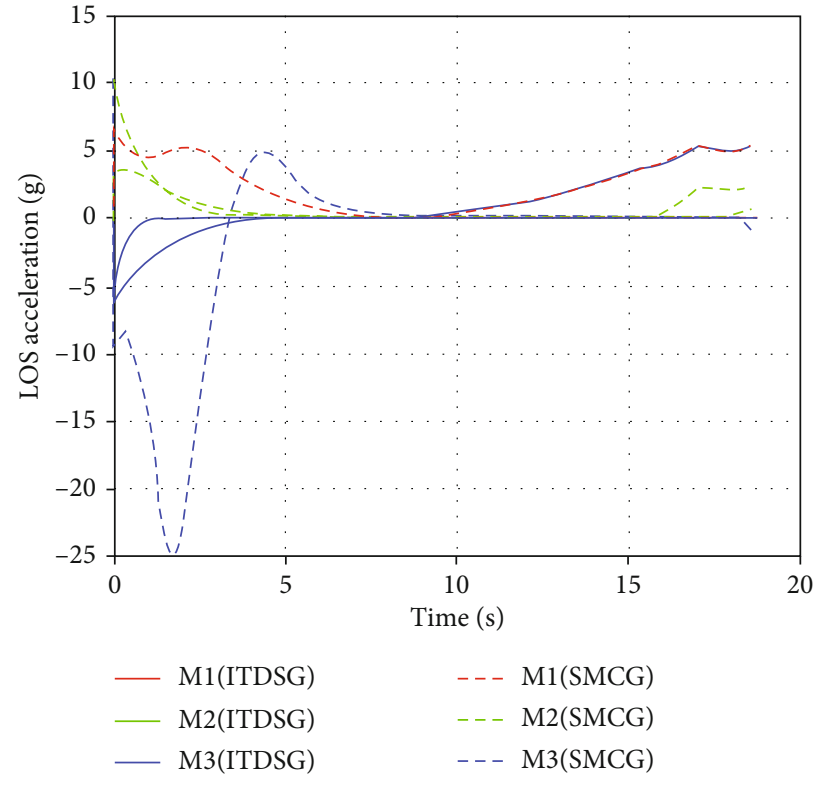

(e) Accelerations on the LOS direction

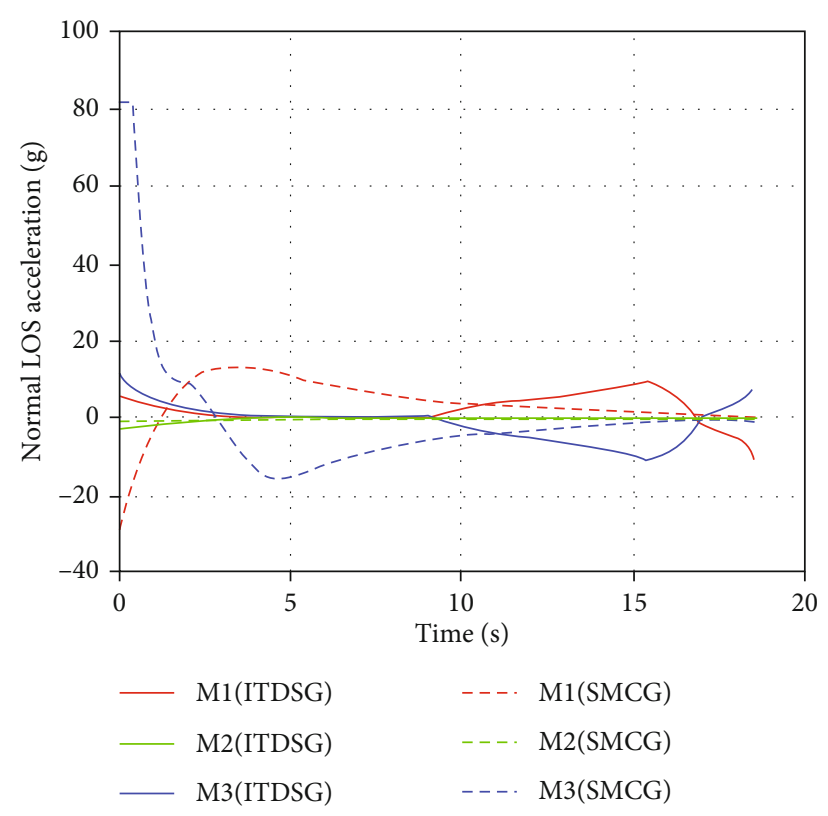

(f) Accelerations on the normal LOS direction

FIGURE 7: Simulation curves for a constant velocity target.

TABLE 2: Energy consumption.

\begin{tabular}{lccccc}
\hline \multirow{2}{*}{$J_{u}$} & \multirow{2}{*}{ Missile } & \multicolumn{2}{c}{ Case 1 } & \multicolumn{2}{c}{ Case 2 } \\
& & ITDSG & SMCG & ITDSG & SMCG \\
\hline \multirow{3}{*}{$J_{u r}\left(\mathrm{~m} / \mathrm{s}^{2}\right)$} & Missile 1 & 254.4 & 220.4 & 314.5 & 1025.4 \\
& Missile 2 & 133.9 & 86.5 & 217.9 & 167.8 \\
& Missile 3 & 222.5 & 565.9 & 504.6 & 548.4 \\
\hline \multirow{3}{*}{$J_{u q}\left(\mathrm{~m} / \mathrm{s}^{2}\right)$} & Missile 1 & 553.9 & 1024.7 & 1021.5 & 1858.7 \\
& Missile 2 & 53.7 & 41.8 & 944.9 & 911.1 \\
& Missile 3 & 647.3 & 4605.3 & 1076.7 & 1740.4 \\
\hline
\end{tabular}

$$
\begin{aligned}
\dot{\mathbf{V}}_{1}= & x_{1 i v} \dot{x}_{1 i v}+x_{2 i v} \dot{x}_{2 i v}=x_{1 i v}\left(\frac{r_{i v}^{2}}{\dot{r}_{i v}^{2}} x_{3 i v}^{2}+\widehat{d}_{r i v}-\frac{r_{i v}}{\dot{r}_{i v}^{2}} u_{r i}\right) \\
& +x_{2 i v}\left(-2 \frac{r_{i v}}{\dot{r}_{i v}} x_{3 i v}+\widehat{d}_{q i v}-\frac{1}{r_{i v}} u_{q i}\right) \\
= & x_{1 i v}\left(\psi_{1}(t)+\eta_{1 i} \operatorname{sign}\left(x_{1 i v}\right)-\widehat{d}_{r i v}+d_{r i v}\right) x_{1 i v} \\
& +x_{2 i v}\left(\psi_{2}(t)+\eta_{2 i} \operatorname{sign}\left(x_{2 i v}\right)-\widehat{d}_{q i v}+d_{q i v}\right) x_{2 i v}
\end{aligned}
$$

where $\psi_{1}(t)=-\left(k_{11 i}+k_{12 i}(\mu(t) / \mu(t))\right) \$$ and $\$ \psi_{2}(t)=-\left(k_{21 i}\right.$ $\left.+k_{22 i}(\mu(t) / \mu(t))\right)$.

Since $\left|-\widehat{d}_{\text {riv }}+d_{\text {riv }}\right| \leq \eta_{1 i},\left|-\widehat{d}_{q i v}+d_{q i v}\right| \leq \eta_{2 i} ;$ then, we have

$$
\left.\dot{\mathbf{V}}_{1} \leq \psi_{1}(t) x_{1 i v}^{2}-\psi_{2}(t)\right) x_{2 i v}^{2} \leq-\left(\lambda_{\min 1}+\lambda_{\min 2} \frac{\dot{\mu}(t)}{\mu(t)}\right) \mathbf{V}_{1} \text {, }
$$

where $\lambda_{\min 1}=\min \left\{k_{11 i}, k_{21 i}\right\}$ and $\lambda_{\min 2}=\min \left\{k_{12 i}, k_{22 i}\right\}$. Then, according to Lemma 3 , we can obtain that the state variables $x_{1 i v}$ and $x_{2 i v}$ gradually converge to zero in a prescribed-time T. According to the definition of $x_{1 i v}$ and $x_{2 i v}$, it is clear to see that all the $t_{g o f i}$ can converge to $T_{g \circ f}^{*}$, and all the $\dot{q}_{i}$ converge to zero in a prescribed-time $T$, respectively. Besides, the $T_{\text {gof }}^{*}$ can converge to a same value in a prescribed-time $T$ by applying consensus protocol Eq. (10), which has been proved in Lemma 2.

Remark 8 . The convergence speed of the system states is controllable with the proposed guidance law in this paper. From Eq. (21), it can be seen that the time for the virtual target to approach the real target is determined by $T_{e}$. By applying the prescribed-time convergence protocol, the desired flight time $T_{g o f i}$ of the missiles converge to a same value at time $T$. In addition, by introducing a time-varying scaling function, the convergence time of the LOS angular rates $\dot{q}_{i}$ and the times-to-go $T_{\text {goi }}$ of the missiles are also determined by the prescribed-time $T$. In other words, by setting different values of $T_{e}$ and $T$, the convergence speed of multimissile cooperative attack a highly maneuvering target can be set arbitrarily.

\section{Simulation}

To illustrate the effectiveness and superiority of the proposed ITDSG, we design two groups of simulations in the scenario of three missiles cooperative attacking a target with constant velocity and highly maneuvering target, respectively. Simulations with the slide mode cooperative guidance law (SMCG) in [21] are carried out for comparison.

4.1. Case 1: Constant Velocity Target. Let us consider the scenario of three missiles attacking single target from different directions; the first group simulations are performed to verify 


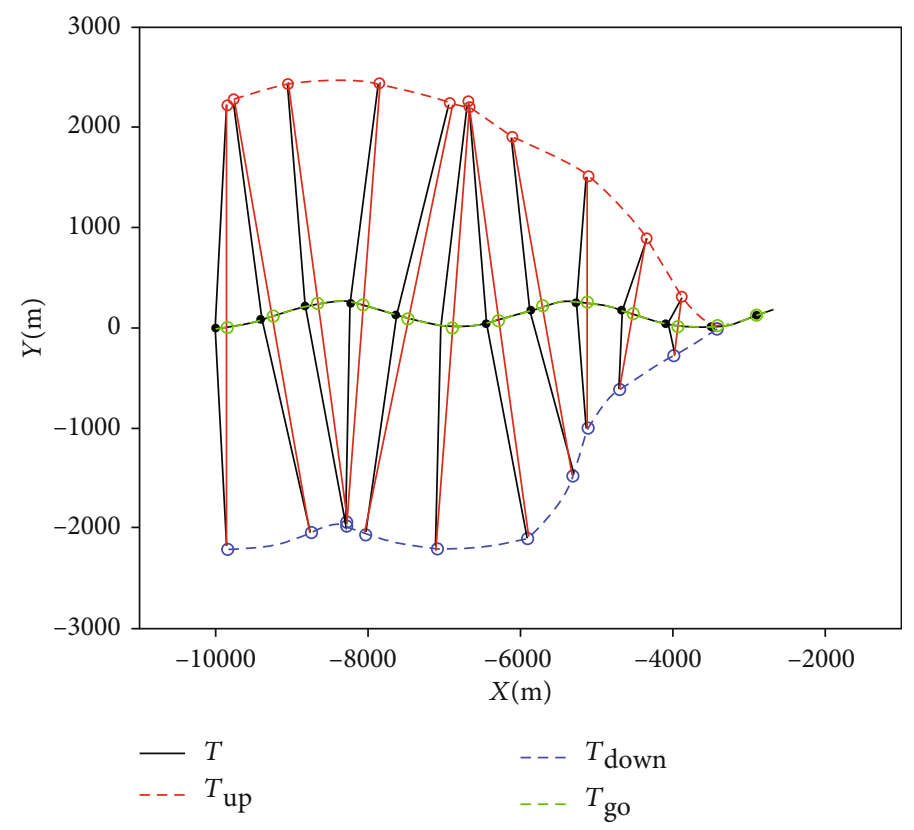

FIgURE 8: Real and virtual targets with high maneuvering.

the performances of the proposed ITDSG compared with SMCG.

Table 1 shows the simulation initial conditions of three missiles. Suppose that the target has a constant velocity, and the initial position of the target is $(-10000 \mathrm{~m}, 0 \mathrm{~m})$, the initial flight path angle of the target is $\theta T 0=0^{\circ}$.

Assume that the communication network graph of three missiles is connected and undirected, Figure 5 shows the communication typology between the three missiles.

The ESO parameters in LOS and normal LOS direction are the same; they are set as $\gamma_{11}=200, \gamma_{12}=300, \delta=0.01$, and $\mu=0.5$. The parameters of ITDSG are set as $k_{11 i}=1$, $k_{12 i}=1, k_{21 i}=0.5, k_{22 i}=1, \eta_{1 i}=0.1$, and $\eta_{2 i}=0.05$; besides, the parameters of $\mu(t)$ are set as $h=2$ and $T=19$; what is more, there are two parameters for calculation of virtual targets $T_{p \text { max }}=8$ and $\delta_{t}=1.5$. In order to compare SMCG with ITDSG under the same conditions, we set the desired LOS angles of SMCG as the same with the terminal LOS angles $q_{f i}(i=1,2,3)$ of ITDSG.

Note 1. Since the energy of the missile is limited, so the smaller energy needs to provide by the guidance law is, the more favorable application the guidance law is. Therefore, an energy consumption evaluation indicators are defined as Eq. (35).

$$
\left\{\begin{array}{l}
J_{u r}=\int_{0}^{t}\left|u_{r}(t)\right| d(t), \\
J_{u q}=\int_{0}^{t}\left|u_{q}(t)\right| d(t) .
\end{array}\right.
$$

Figure 6 shows the trajectories of the real target and virtual targets, where $T$ denotes the real target and $T_{\text {up }}, T_{\text {down }}$, and $T_{\text {go }}$ denote the virtual target from different directions. Figure 7 shows the comparative simulation results of three missiles against a target with SMCG and ITDSG methods, in the scenario of three missiles cooperatively hitting a constant velocity target. Further, Figure 6 shows the trajectories of real target and virtual targets; in ITDSG, all missiles aim the virtual targets. It is clear to see that all the three virtual targets gradually approach the real target.

The effectiveness of the proposed ITDSG against a constant velocity target can be verified as follows: Figures 7(a)7 (c) show that all of the missiles can hit the target simultaneously, which indicates that the proposed ITDSG subject to the decoupled model is effective for the time consistency. Figure 7(c) shows that all missiles with ITDSG approach the real target from different directions, which shows that the proposed dynamic surrounding attack strategy and the cooperative guidance law are effective.

In terms of missile trajectories, ITDSG has a better performance than SMCG in Figure 7(a). In the initial stage, the trajectories of ITDSG are smoother than SMCG; then, in the final stage, the trajectories are similar. The reason is that when applying SMCG, there are bigger errors between the initial LOS angles and desired angles of each missile; in order to decrease the errors, larger lateral accelerations must be generated to change the missile's flight path to meet the constraint of LOS angles; but when applying the proposed ITDSG strategy, all the missiles aim at the virtual targets, and there are no LOS angle constrains; thus, the missile's trajectories are relatively smooth. With the same reason, Figure 7(f) shows that in the initial stage the control inputs $u_{q}$ in SMCG are bigger than in ITDSG. In the whole guidance process, the energy consumption in ITDSG is also smaller than in SMCG that is listed in Table 2.

In terms of the impact time, the SMCG and ITDSG have the same performance. From the simulation curves in 


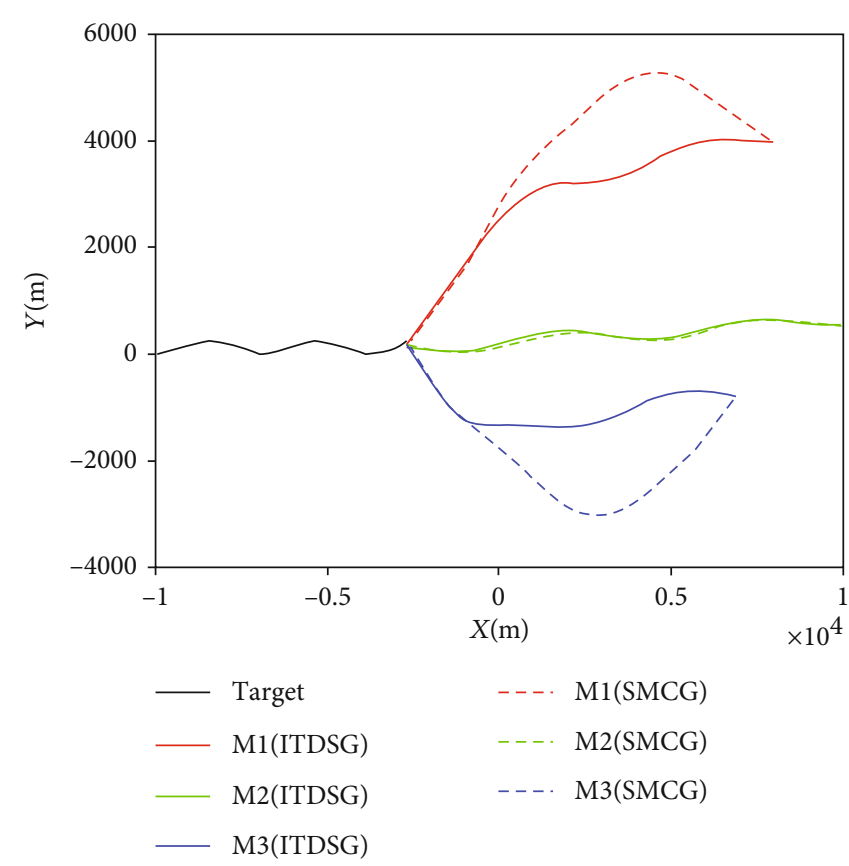

(a) Trajectories for missiles and target

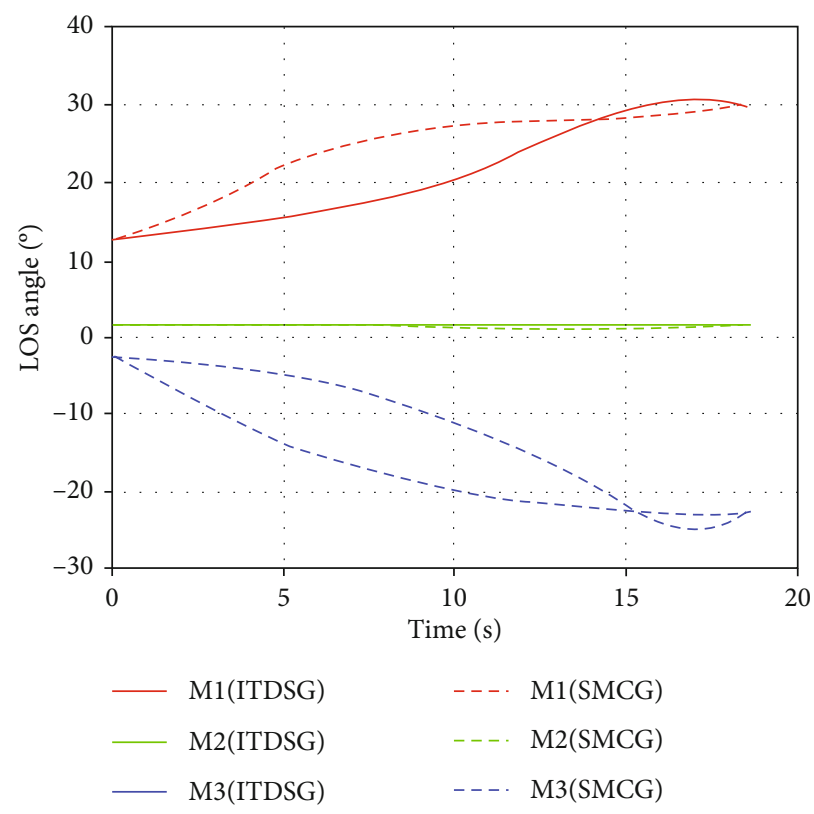

(c) LOS angles for missiles

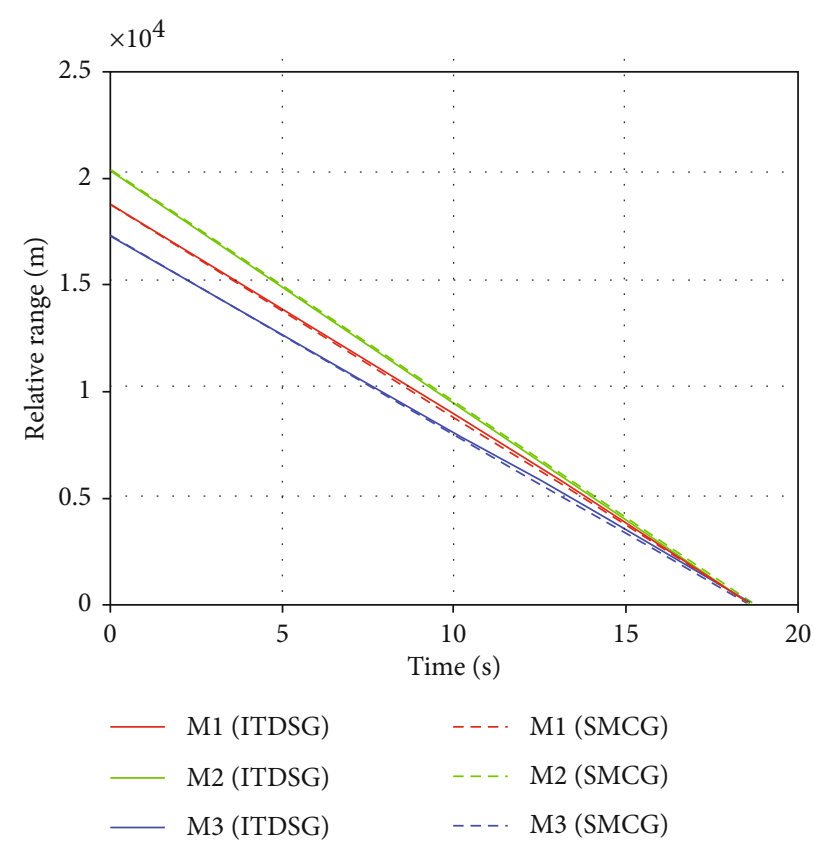

(b) Distance between missiles and target

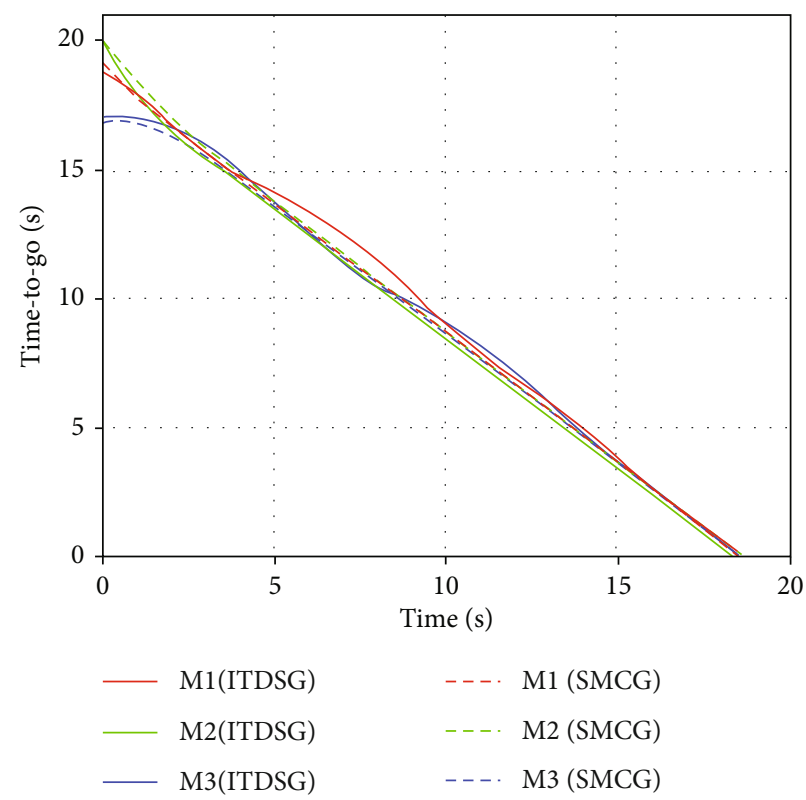

(d) Time-to-go for missiles

FIgURe 9: Continued. 


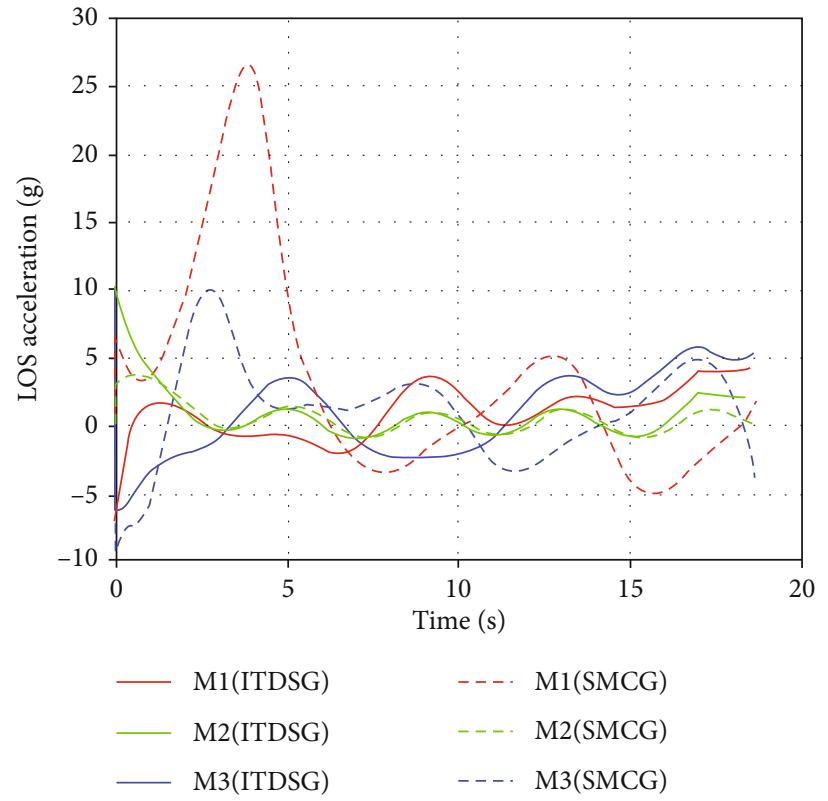

(e) Accelerations on the LOS direction

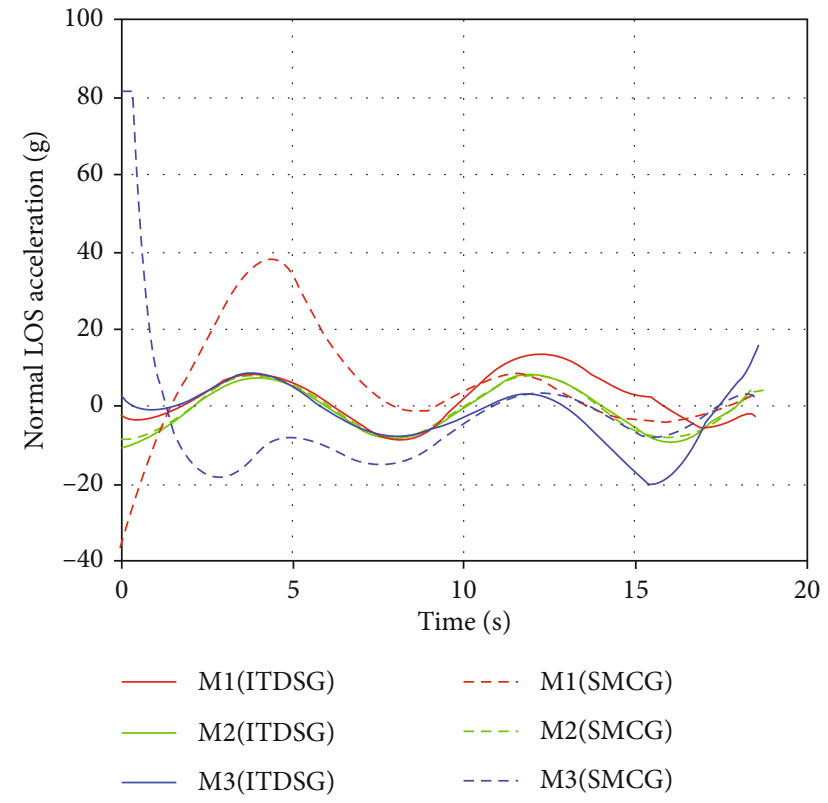

(f) Accelerations on the normal LOS direction

FIGURE 9: Simulation curves for a highly maneuvering target.

Figure $7(\mathrm{~d})$, it is clear that even though the missile initial values of $t_{g o i}$ are different, they can converge to the same value under the action of $u_{r i}$, which indicates that the SMCG and the proposed ITDSG law subject to the decoupled model with ITDSG strategy are both effectiveness in impact time. Besides, in Figure 7(e), bigger accelerations in the LOS direction are generated because of the bigger errors between the times-to-go and their desired values of different missiles. What is more, Figures $7(\mathrm{e})$ and $7(\mathrm{f})$ show clearly that there are small fluctuations of acceleration curves in LOS and normal LOS directions with ITDSG after 9 seconds, which is caused by the maneuver of virtual target approach the real target.

As a result, in the scenario of intercepting a constant velocity target, ITDSG and SMCG both show good performance. Because ITDSG uses a dynamic surrounding attack strategy without LOS angle constraint, the trajectories by ITDSG are smoother than that by SMCG; the accelerations in normal LOS directions are also smaller.

4.2. Case 2: Highly Maneuvering Target. In this case, the performance of the proposed ITDSG is investigated in the scenario of three missiles attacking single highly maneuvering target from different directions.

In order to further illustrate the superiority of ITDSG over SMCG, simulations are conducted with the same cooperative guidance laws and the same missile parameters as those in Case 1, the only difference is that the target maneuver with a bigger overload $n_{t}=8 g \sin ((\pi / 4) t)$, where $g=9.8$ $\mathrm{m} / \mathrm{s}^{2}$. Furthermore, the selection principle of $q_{f i}(i=1,2,3)$ in SMCG is the same as in Case 1. Figure 8 shows the curves of virtual targets with highly maneuvering target subject to the real target motion.

Similar to Case 1, Figure 8 shows the trajectories of the real target and virtual targets, where $T$ denotes the real target and $T_{\text {up }}, T_{\text {down }}$, and $T_{\text {go }}$ denote the virtual target from different directions; Figure 9 shows the effectiveness of the proposed ITDSG strategy against a highly maneuvering target. Figures 9(a) and 9(c) show that the proposed dynamic surrounding attack strategy is effective; three missiles can intercept the target from different directions; Figures 7(a), $7(\mathrm{~d})$, and 7(b) show that the proposed ITDSG is also effective for the time consistency.

Similar to the analysis in Case 1, Figure 9(a) shows that trajectories of ITDSG are smoother than SMCG; Figure 9(f) and Table 2 show that the maximum overloads in LOS and normal LOS directions of ITDSG are smaller than SMCG, and the total control energy of ITDSG is also smaller. The time constraint performance of the ITDSG is the same as SMCG, which shows in Figures 9(b) and 9(d). What is more, the difference between Case 1 and Case 2 is that the acceleration curves of all missiles in the two directions can both converge but not zero due to the target maneuver.

As a result, the proposed guidance law ITDSG can be suitable for constant velocity speed as well as highly maneuvering target. Besides, by introducing virtual targets instead of impact LOS angle to achieve dynamic surrounding attacks, ITDSG has better performance in trajectories and energy consumption than SMCG.

\section{Conclusion}

In this paper, we developed a new dynamic surrounding attack cooperative guidance law against highly maneuvering target without a preset LOS angle constraint. Firstly, we proposed the strategy of dynamic surrounding attack by introducing virtual targets and then established the cooperative guidance models between multiple missiles and multiple virtual targets. Finally, by using the dynamic inverse method to decouple the coupled cooperative guidance model, and the 
ITDSG subject to the decoupled model is designed based a prescribed-time stable method. Additionally, ESOs are introduced to estimate the disturbances in the LOS and normal LOS directions. In order to demonstrate the effectiveness and superiority of the proposed ITDSG, two groups of comparison simulations are carried out with SMCG in the scenarios of three missiles cooperative attack a constant velocity or highly maneuvering target.

Compared with the existing works, the advantages of the proposed ITDSG are that it does not require to set desired LOS angles in advance, and the couples in the cooperative guidance model are also considered. The limitation of the proposed guidance law in this paper lies in the need for precise target information, such as $q, \dot{q}, r$, and $\dot{r}$. In the future work, we would develop a novel cooperative guidance law with dynamic surrounding attack when there are seeker measurement errors.

\section{Data Availability}

The data used to support the findings of this study are included within the article.

\section{Conflicts of Interest}

The authors declared that they have no conflicts of interest to this work.

\section{Acknowledgments}

Thanks are due to the financial support provided by the National Natural Science Foundation of China (Grant No. 61973253), the Aviation Science Foundation of China (20180153001), and the Foundation of National Defense Science and Technology Key Laboratory (6142219180202).

\section{References}

[1] N. Balhance, M. Weiss, and T. Shima, "Cooperative guidance law for intrasalvo tracking," Journal of Guidance, Control, and Dynamics, vol. 40, no. 6, pp. 1441-1456, 2017.

[2] G. Li, Y. Wu, and P. Xu, "Adaptive fault-tolerant cooperative guidance law for simultaneous arrival," Aerospace Science and Technology, vol. 82-83, pp. 243-251, 2018.

[3] X. Ai, L. Wang, J. Yu, and Y. Shen, "Field-of-view constrained two-stage guidance law design for three-dimensional salvo attack of multiple missiles via an optimal control approach," Aerospace Science and Technology, vol. 85, pp. 334-346, 2019.

[4] I. Jeon and J. Lee, "Homing guidance law for cooperative attack of multiple missiles," Journal of Guidance Control and Dynamics, vol. 33, no. 1, pp. 275-280, 2010.

[5] M. Tahk, S. Shim, S. Hong, H. Choi, and C. Lee, "Impact time control based on time-to-go prediction for sea-skimming antiship missiles," IEEE Transactions on Aerospace and Electronic Systems, vol. 54, no. 4, pp. 2043-2052, 2018.

[6] J. L. Zhou, J. Y. Yang, and Z. K. Li, "Simultaneous attack of a stationary target using multiple missiles: a consensus-based approach," Science China Information Sciences, vol. 60, no. 7, 2017.
[7] J. Zhao, S. Zhou, and R. Zhou, "Distributed time-constrained guidance using nonlinear model predictive control," Nonlinear Dynamics, vol. 84, no. 3, pp. 1399-1416, 2016.

[8] Q. ZHAO, X. DONG, Z. LIANG, C. BAI, J. CHEN, and Z. REN, "Distributed cooperative guidance for multiple missiles with fixed and switching communication topologies," Chinese Journal of Aeronautics, vol. 30, no. 4, pp. 1570-1581, 2017.

[9] J. B. ZHAO and S. YANG, "Integrated cooperative guidance framework and cooperative guidance law for multi-missile," Chinese Journal of Aeronautics, vol. 31, no. 3, pp. 546-555, 2018.

[10] J. Zhou and J. Yang, "Distributed guidance law design for cooperative simultaneous attacks with multiple missiles," Journal of Guidance Control and Dynamics, vol. 39, no. 10, pp. 2436-2444, 2016.

[11] S. Kang, J. N. Wang, G. Li, J. Y. Shan, and I. R. Petersen, "Optimal cooperative guidance law for salvo attack: an MPC-based consensus perspective," IEEE Transactions on Aerospace and Electronic Systems, vol. 54, no. 5, pp. 23972410, 2018.

[12] M. Mozaffari, B. Safarinejadian, and T. Binazadeh, "Optimal guidance law based on virtual sliding target," Journal of Aerospace Engineering, vol. 30, no. 3, pp. 1-11, 2017.

[13] X. Wei, Y. Wang, S. Dong, and L. Liu, “A three-dimensional cooperative guidance law of multi-missile system," International Journal of Aerospace Engineering, vol. 2015, 8 pages, 2015.

[14] Z. Yang, H. Wang, D. Lin, and L. Zang, "A new impact time and angle control guidance law for stationary and nonmaneuvering targets," International Journal of Aerospace Engineering, vol. 2016, 14 pages, 2016.

[15] Y. Li, H. Zhou, and W. Chen, "Three-dimensional impact time and angle control guidance based on MPSP," International Journal of Aerospace Engineering, vol. 2019, 16 pages, 2019.

[16] E. Zhao, T. Chao, S. Wang, and M. Yang, "Multiple flight vehicles cooperative guidance law based on extended state observer and finite time consensus theory," Proceedings of the Institution of Mechanical Engineers, Part G: Journal of Aerospace Engineering, vol. 232, no. 2, pp. 270-279, 2016.

[17] W. Zou, P. Shi, Z. Xiang, and Y. Shi, "Finite-time consensus of second-order switched nonlinear multi-agent systems," IEEE Transactions on Neural Networks and Learning Systems, vol. 31, no. 5, pp. 1757-1762, 2020.

[18] W. Zou, C. K. Ahn, and Z. Xiang, "Fuzzy-approximationbased distributed fault-tolerant consensus for heterogeneous switched nonlinear multiagent systems," IEEE Transactions on Fuzzy Systems, vol. 99, pp. 1-10, 2021.

[19] M. Nikusokhan and H. Nobahari, "Closed-form optimal cooperative guidance law against random step maneuver," IEEE Transactions on Aerospace Electronic Systems, vol. 52, no. 1, pp. 319-336, 2016.

[20] W. Su, K. Li, and L. Chen, "Coverage-based three-dimensional cooperative guidance strategy against highly maneuvering target," Aerospace Science and Technology, vol. 85, pp. 556-566, 2019.

[21] J. Song, S. Song, and S. Xu, "Three-dimensional cooperative guidance law for multiple missiles with finite- time convergence," Aerospace Science and Technology, vol. 67, pp. 193205, 2017. 
[22] X. Wang and X. Lu, "Three-dimensional impact angle constrained distributed guidance law design for cooperative attacks," ISA Transactions, vol. 73, pp. 79-90, 2018.

[23] S. He, D. Lin, and J. Wang, "Robust terminal angle constraint guidance law with autopilot lag for intercepting maneuvering targets," Nonlinear Dynamics, vol. 81, no. 1-2, pp. 881-892, 2015.

[24] Q. Chen, X. Wang, J. Yang, and Z. Wang, "Trajectory-following guidance based on a virtual target and an angle constraint," Aerospace Science and Technology, vol. 87, pp. 448-458, 2019.

[25] Q. Hu, T. Han, and M. Xin, “Three-dimensional guidance for various target motions with terminal angle constraints using twisting control," IEEE Transactions on Industrial Electronics, vol. 67, no. 2, pp. 1242-1253, 2020.

[26] J. Lee, I. Jeon, and M. Tahk, "Guidance law to control impact time and angle," IEEE Transactions on Aerospace and Electronic Systems, vol. 43, no. 1, pp. 301-310, 2007.

[27] Y. Zhang, G. Ma, and A. Liu, "Guidance law with impact time and impact angle constraints," Chinese Journal of Aeronautics, vol. 26, no. 4, pp. 960-966, 2013.

[28] N. Harl and S. N. Balakrishnan, "Impact time and angle guidance with sliding mode control," IEEE Transactions on Control Systems Technology, vol. 20, no. 6, pp. 1436-1449, 2012.

[29] X. Wang, Y. Zhang, and H. Wu, "Distributed cooperative guidance of multiple anti-ship missiles with arbitrary impact angle constraint," Aerospace Science and Technology, vol. 46, pp. 299-311, 2015.

[30] L. Jing, L. Zhang, J. Guo, and N. Cui, "Fixed-time cooperative guidance law with angle constraint for multiple missiles against maneuvering target," IEEE Access, vol. 99, p. 1, 2020.

[31] Y. Wang, Y. Song, D. J. Hill, and M. Krstic, "Prescribed-time consensus and containment control of networked multiagent systems," IEEE Transactions on Cybernetics, vol. 49, no. 4, pp. 1138-1147, 2019.

[32] Y. Ren, W. Zhou, Z. Li, L. Liu, and Y. Sun, "Prescribed-time cluster lag consensus control for second-order non-linear leader-following multiagent systems," ISA Transactions, vol. 109, pp. 49-60, 2020.

[33] J. Han, "From PID to active disturbance rejection control," IEEE Transactions on Industrial Electronics, vol. 56, no. 3, pp. 900-906, 2009. 